\title{
Zentralvorstandssitzung vom 10. Januar 2013
}

\begin{abstract}
Masterplan Hausarztmedizin - Für die Neuorganisation von TARMED Suisse soll eine Taskforce Lösungen bis zum 30. April 2013 ausarbeiten. Aufgrund der neuen Ausgangslage beschliesst der Zentralvorstand (ZV), für das Teilprojekt des Masterplans «Finanzierung und Versorgung» die Priorisierung von Kapitel 40 und die Übergangslösung mit Roadmap nochmals in der Delegiertenversammlung (DV) zu diskutieren und das Gespräch mit Hausärzte Schweiz zu suchen.

Roadmap nachhaltiges Gesundheitssystem - Die SAMW lädt die FMH dazu ein, bis Mitte April ihre Kompetenzen und Positionen in die Roadmap «Ein nachhaltiges Gesundheitssystem für die Schweiz»
\end{abstract}

einzubringen. Auf Wunsch des ZV sollen alle Ressorts bis Ende Februar Stellung zur Roadmap nehmen, um die Antworten anschliessend diskutieren zu können.

Ausschluss Doppeltitelträger - In den Hausarztlistenmodellen einiger Krankenkassen werden die Doppeltitelträger ausgeschlossen. Der ZV stellt sich grundsätzlich gegen jegliche Listenmodelle und entscheidet, ein allfälliges Musterverfahren mitzuunterstützen, um Druck gegenüber den Kassen auszuüben. Da die kantonale Ebene zentral ist, soll die FMH auf die KKA zugehen.

\section{Séance du Comité central du 10 janvier 2013}

Masterplan «Médecine de famille» - Concernant la réorganisation de TARMED Suisse, une taskforce a été chargée d'élaborer des solutions d'ici au 30 avril 2013. Dans ce contexte, le Comité central a décidé de rediscuter à l'Assemblée des délégués du sous-projet «Financement et soins de base», et notamment de la question de traiter en priorité le chapitre 40 , ainsi que de la solution transitoire avec la feuille de route, et de chercher le dialogue avec Médecins de famille Suisse.

Feuille de route «Un système de santé durable» L'ASSM invite la FMH à partager ses compétences et à présenter ses positions d'ici à la mi-avril dans le cadre de la feuille de route «Un système de santé durable pour la Suisse». A la demande du Comité central, les domaines d'activité de la FMH ont jusqu'à fin février pour prendre position à ce sujet, afin de pouvoir ensuite en discuter.

Exclusion des porteurs de doubles titres - Certaines caisses-maladie excluent les porteurs de doubles titres de leur liste des médecins de famille. Opposé par principe aux modèles de listes, le Comité central a décidé de soutenir une éventuelle procédure type afin de faire pression sur les caisses. Comme la compétence revient aux cantons, la FMH prendra contact avec la Conférence des sociétés cantonales de médecine. 